\title{
Inventaire archéologique à Hiva Oa (Marquises)
}

\section{Catherine Chavaillon et Éric Olivier}

\section{(2) OpenEdition}

Journals

Édition électronique

URL : http://journals.openedition.org/jso/444

DOI : $10.4000 /$ jso.444

ISSN : $1760-7256$

Éditeur

Société des océanistes

Édition imprimée

Date de publication : 1 décembre 2005

Pagination : 157-171

ISSN : 0300-953x

\section{Référence électronique}

Catherine Chavaillon et Éric Olivier, «Inventaire archéologique à Hiva Oa (Marquises) », Journal de la

Société des Océanistes [En ligne], 120-121 | Année 2005, mis en ligne le 27 novembre 2008, consulté le 01 mai 2019. URL : http://journals.openedition.org/jso/444 ; DOI : 10.4000/jso.444 


\title{
Inventaire archéologique à Hiva Oa (Marquises)
}

par

\author{
Catherine CHAVAILLON* et Éric OLIVIER**
}

\section{RÉSUMÉ}

L'inventaire archéologique de l'île de Hiva Oa a été commandité par le service de la Culture et du Patrimoine de Polynésie française. Cette île, autrefois très peuplée, a subi une dépopulation massive à la fin $d u X I X^{e}$ siècle. Les survivants se sont groupés dans un petit nombre de villages situés en bord de mer autour des premières missions (catholiques et protestantes). Les vestiges des anciens aménagements des vallées ont été abandonnés à la forêt et à l'usure du temps. De nombreux objets (dalles sculptées, statues, pétroglyphes, pierres à cupules et aiguisoirs) sont restés en place. Notre objectif est de répertorier ces pièces dans leur contexte archéologique et de tenter de recomposer, sur la carte de l'île, l'organisation du paysage ancien de certaines vallées (les grands sites cérémoniels, les habitations dispersées sur les pentes aux alentours, les terrasses de cultures, les fosses silos, les me'ae ${ }^{1}$ et les sites défensifs) avant que ces témoins ne disparaissent complètement. Les statues et dalles sculptées sont taillées, pour la plupart, dans un tuf volcanique tendre, sensible à l'érosion et aux dégradations d'origine végétale, animale ou humaine.

Cet inventaire est encore très incomplet, mais il s'enrichit au fil du temps à travers les prospections et les relevés des sites de secteurs encore inexplorés, trop rapidement aperçus ou menacés de destruction à court terme. Il est urgent de gérer au mieux ce patrimoine marquisien afin qu'il trouve la place qu'il mérite au sein du patrimoine mondial.

MoTS-CLÉS : Inventaire archéologique, patrimoine, archipel des Marquises.

\section{ABSTRACT}

The French Polynesian Service of Culture and Heritage has been financing the archeological inventory of the island of Hiva Oa. Once densely populated, this island suffered a huge depopulation at the end of the nineteenth century. The survivors regrouped in a small number of coastal villages around the first (catholic and protestant) missions. What remained of the original developments was abandoned to the forest and weathering. Numerous objects (sculptured slab stones, statues, petroglyphs, cup stones and sharpening stones) can be found in situ. Our objective is to catalogue these pieces in their archeological context and to try and reconfigure, on a map of the island, the organization of the former landscape of specific valleys (the great ceremonial sites, the scattered dwellings on the neighbouring slopes, the cultivated terrasses, the silos pits, the meae, and the defensive sites) before this body of evidence completely disappears. In particular, the statues and sculptured stones are, in the main, cut out of tender volcanic tuff which is sensitive to erosion and to plant, animal andlor human degradation.

This inventory, still in the making, expands through the prospections and site-surveys of sections as yet unexplored, overlooked or threatened with short- term destruction.

We must urgently manage our Marquesan heritage as best as we can so that it finds the place it deserves in the world heritage.

KEYWORDS: archeological inventory, heritage, Marquesas archipelago.

1. Le mot me'ae représente tout lieu sacré (tapu), qu'il soit construit ou laissé naturel. D'après Pierre Ottino, il s'applique avant tout à un site funéraire. Chaque famille en possédait un qui peut, dans certains cas, désigner l'endroit où l'on traitait le

* Prestataire au service de la Culture et du Patrimoine de Polynésie française, cathchavaillon@mail.pf

** Photographe et professeur de mathématiques et d'informatique au collège Ste-Anne, à Atuona, Hiva Oa. 


\section{Situation géographique de Hiva Oa}

L'archipel des Marquises est constitué de douze îles et îlots répartis en deux groupes comprenant chacun trois îles habitées. Dans le groupe nord-ouest, Ua Pou et Ua Huka se trouvent de part et d'autre de la capitale administrative Nuku Hiva. Hiva Oa est l'île principale du groupe sud-est, Tahuata n'est séparée d'elle que par le canal du Bordelais, Fatu Hiva en est éloignée de $75 \mathrm{~km}$ au sud-est. Hiva Oa est longue de $40 \mathrm{~km}$ d'Est en Ouest et large d'une dizaine de kilomètres.

Une grande crête dorsale, orientée est sudouest dont les sommets culminent à plus de mille mètres, sépare l'île en quatre parties. La partie sud comprend de nombreuses vallées dont plusieurs bénéficient de rivières permanentes ; certaines, très vastes, ont permis l'installation d'une population importante, de nombreux vestiges en témoignent. Dans la partie nord-est, la vallée de Puamau, connue par la présence des plus grands $t i k i^{2}$ des Marquises, contient la plus remarquable concentration de sculptures de l'île, Eiaone et Hanapaaoa sont aussi très riches de vestiges. La partie nord-ouest, en dehors de Hanaiapa qui possède de l'eau en abondance, est beaucoup plus sèche et plus difficilement accessible. Les vallées sont étroites jusqu'à la grande faille qui s'ouvre à l'océan par la baie de Hanamenu. La partie sud-ouest est plus étroite et pentue, peu de vallées sont habitables. Trois zones de plateaux intérieurs, l'une à l'Ouest entre Hanamenu et Taaoa, une autre au centre de l'île au Nord de l'aéroport et la troisième au-dessus de Hanaiapa et Puamau, recèlent apparemment peu de groupes de structures organisées. La côte est fortement découpée, les récifs coralliens sont presque inexistants, les plages sont le plus souvent étroites et couvertes de galets, quelques hautes falaises atteignant 200 et $300 \mathrm{~m}$ plongent dans l'océan.

\section{Physionomie archéologique de l'île}

La vague de mortalité dévastatrice de la fin du $\mathrm{XIX}^{\mathrm{e}}$ au début $\mathrm{du} \mathrm{Xx}^{\mathrm{e}}$ siècle, conséquence du contact avec les navigateurs occidentaux, a rassemblé la plupart des survivants en bord de mer dans les vallées principales. Les longues périodes de sécheresse et le marasme économique ont vidé peu à peu les vallées de leurs habitants qui, lorsqu'ils n'ont pas quitté l'île pour vivre à Tahiti, résident maintenant à Taaoa, Atuona, Puamau, Hanapaaoa ou Hanaiapa. Quelques vallées éloignées conservent des habitations actuelles, le plus souvent utilisées d'une façon temporaire. Les grand sites de moyenne et de haute vallée, devenus parfois plantations à la fin du XIX ${ }^{\mathrm{e}}$ et début du $\mathrm{XX}^{\mathrm{e}}$ siècle, puis territoires de chasse, sont encore présents sous leur linceul de végétation et couvrent une grande partie de l'île.

Il reste généralement peu de choses des sites construits à l'arrière des plages. Ils ont été démolis, recouverts d'alluvions ou immergés par les raz de marée et les crues, ou détruits par les aménagements urbains. C'est en moyenne vallée que sont groupés la plupart des grands sites cérémoniels, entourés de paepae ${ }^{3}$ et de lieux d'habitation clairsemés. Les berges des torrents sont consolidées par des enrochements, certains sentiers gardent des traces d'empierrement, les terrasses de cultures, enclos, fosses et $u a \mathrm{ma}^{4}$ sont encore lisibles. Les me'ae construits en bordure de rivière sont généralement placés entre celle-ci et son affluent, ou sur une éminence. Sur les pentes des hautes vallées, la plupart des ensembles de structures, souvent de petites dimensions, sont composées de différents niveaux disposés en U ou en escalier. Les crêtes couvertes de fougères sont parfois percées de fosses aux parois verticales, quelques-unes en forme de bouteille, leurs épaulements sont quelquefois nivelés pour édifier un méae, un site défensif ou réserver un espace de refuge. Les zones de plateaux ne mon-

corps du défunt : le taha tupapau. «Le mot me'ae désigne plus habituellement le lieu sacré d'une tribu ou d'une vallée, dans lequel reposent les vestiges des ancêtres les plus prestigieux. Il peut être très proche ou même faire partie d'un tohua, cependant le me'ae le plus tapu en était dissocié. Il comprend un ensemble de plates-formes et d'autres structures moins lisibles : murets, enclos, pavages etc. Un des traits particuliers de ces me'ae des Marquises est, si l'on peut dire, l'absence de plan type. Lors de leur élaboration, on tenait compte de la topographie de l'endroit et l'on respectait les rochers en place. Ces derniers sont parfois ornés de pétroglyphes ou peuvent servir à ancrer une plate-forme. Ces accidents remarquables du terrain, au même titre que le ruisseau tout proche ou le piton rocheux le dominant, font partie intégrante du lieu sacré, qui trouve sa raison d'être en partie par la présence de ces éléments "naturels". » (Ottino et de Berg, 1991 : 34).

2. Le tiki est une représentation du ou d'un ancêtre divinisé. Par extension, tiki devient synonyme d'image, dessin.

3. Paepae, plate-forme de pierre rectangulaire surélevée comportant deux niveaux. La façade du second niveau des paepae les plus soignés est faite de dalles de pierre ou de ke'etu - tuf volcanique - posées sur chant et recouvertes de belles dalles planes ou de galets. Les dalles de ke'etu sont parfois sculptées ou gravées. Selon son utilisation, la plate-forme servira de base à une construction ou non.

4. Ua ma est une fosse silo à usage familial ou communautaire où l'on stockait le ma, la pâte de fruit à pain ou de taro qui se conservait plusieurs années. 
trent pas, d'une façon évidente, les signes d'une occupation permanente, mais leur prospection n'a pas été systématique.

\section{Historique des recherches archéologiques}

Les premiers rapports d'inventaire de l'île datent des années 1920 à 1925. Celui de Clayssen (1921) décrit brièvement six ensembles de structures dans la vallée d'Atuona, deux dans la vallée de Puamau, une dans chacune des vallées de Tahauku et Punaei. Ralph Linton (1925) a répertorié et décrit soixante-quinze sites, ajoutant de nombreux plans et photographies qui restent des témoins précieux de leur état et de l'attitude de la population à leur égard au début $\mathrm{du} \mathrm{xx}^{\mathrm{e}}$ siècle. En septembre 1934, l'expédition La Korrigane chargée de collecter des informations ethnographiques et des objets représentatifs fait trois escales à Hiva $\mathrm{Oa}$ : Atuona, Hanaiapa et Hanamenu - des photographies de certains sites et objets figurent dans Le voyage de La Korrigane dans les mers du sud (Coiffier [éd.], 2001). En 1963, Carlyle Smith (1964) choisit le grand tohua de Pekia (Atuona) comme terrain d'investigations. Plan, photographies, sondages et commentaires sont exposés dans un rapport destiné à l'université du Kansas. En 1965 ont été effectués les relevés et sondages de six petits sites placés à l'arrière de la plage de Atuona par Yosihiko Sinoto et Marimari Kellum (1965). En 19671968, Francis Moeava Peltier (1973) participe à l'expédition archéologique dirigée par Yosihiko Sinoto et publie l'inventaire des structures de la basse vallée de Hanaiapa. François Ollier (1970) en décrit sept. En 1972, Arne Skjolsvold (1972) donne les résultats de la fouille d'une grotte de la vallée Hanapete'o sur la côte nord-ouest. En 1972, Peter Bellwood (1972) publie un rapport d'inventaire de l'ensemble de la vallée de Hanatekua pour le Bishop Museum de Hawaii. En 1985, Edmundo Edwards (1985) décrit le tohua Pehekua et ses structures associées, la même année Sidsel Millerstrom et Claudio Christino (1985) fournissent un rapport d'inventaire des pierres gravées et sculptées situées à l'intérieur et aux alentours des vallées habitées. Une mission dirigée par Maeva Navarro (1986) a été suivie d'un rapport concernant des sondages effectués sur trois sites du bord de mer de la vallée de Hanamenu. En 1991, Pierre Ottino et MarieNoëlle De Berg (1993) ont étudié et réhabilité le site Iipona de Puamau. En 1995, Sidsel Millerstrom et Edmundo Edwards (1995) ont livré les résultats de leur étude des peintures rupestres des grottes de Eiaone. Depuis 2002, le service de la
Culture et du Patrimoine nous a chargés de réaliser l'inventaire des pierres gravées et sculptées de l'île dans leur contexte archéologique. En 2004, Pierre Ottino publie un article concernant les tiki marquisiens.

\section{Un inventaire en devenir}

Afin de mettre en place cet inventaire général, il était nécessaire d'établir une nouvelle numérotation des sites, la plus large et la plus ouverte possible, afin de les placer sur la carte et y ajouter tous ceux qu'il nous reste à découvrir (voir Fig. 1).

Sur un total de soixante-treize bassins versants, dix trop arides et trop pentus comportent probablement très peu ou aucune structure, à part peut-être l'emplacement d'un point de pêche ou quelques sépultures nichées dans les trous des falaises. Sur l'ensemble des soixantetrois autres bassins versants, habitables ne serait-ce que d'une façon temporaire, vingt-cinq contiennent un site ayant fait l'objet d'un rapport de sondages ou d'inventaire, vingt sont connus de nous de façon le plus souvent fragmentaire, dix-huit n'ont pas encore été visités. Diviser l'île en bassins versants numérotés permet de les retrouver facilement, car les noms des baies commençant généralement par hana (baie, crique) sont peu discriminants et ne concernent souvent que la zone côtière, les terres de moyenne et de haute vallée portant des noms différents. Cela permet aussi d'intégrer les plateaux des hautes vallées et les points de pêche. Les constructions situées sur les crêtes séparant deux bassins versants portent le numéro de celui avec lequel les communications paraissent les plus probables. À partir du numéro du bassin versant, les sites ont été numérotés dans un ordre géographique en commençant souvent par le site le plus remarquable. Les ensembles de structures, qui se trouvent dans le même secteur et qui paraissent en relation, reçoivent un numéro supplémentaire, ce qui permet de situer les constructions associées ou secondaires qui aideront à l'interprétation. Une lettre majuscule a été ajoutée pour situer chaque structure et une lettre minuscule désigne l'objet.

Les cent vingt-quatre sites rencontrés à ce jour lors de nos excursions et, parmi eux, de grands sites tels que Faepoto (Hanatea B13-01) qui contient seize ensembles de structures, ont trouvé leur place sur la carte. La même attention n'a pu être portée à tous, faute de temps. Certains ensembles ont bénéficié de relevés de surface précis, d'un plan triangulé (sans théodolite), 


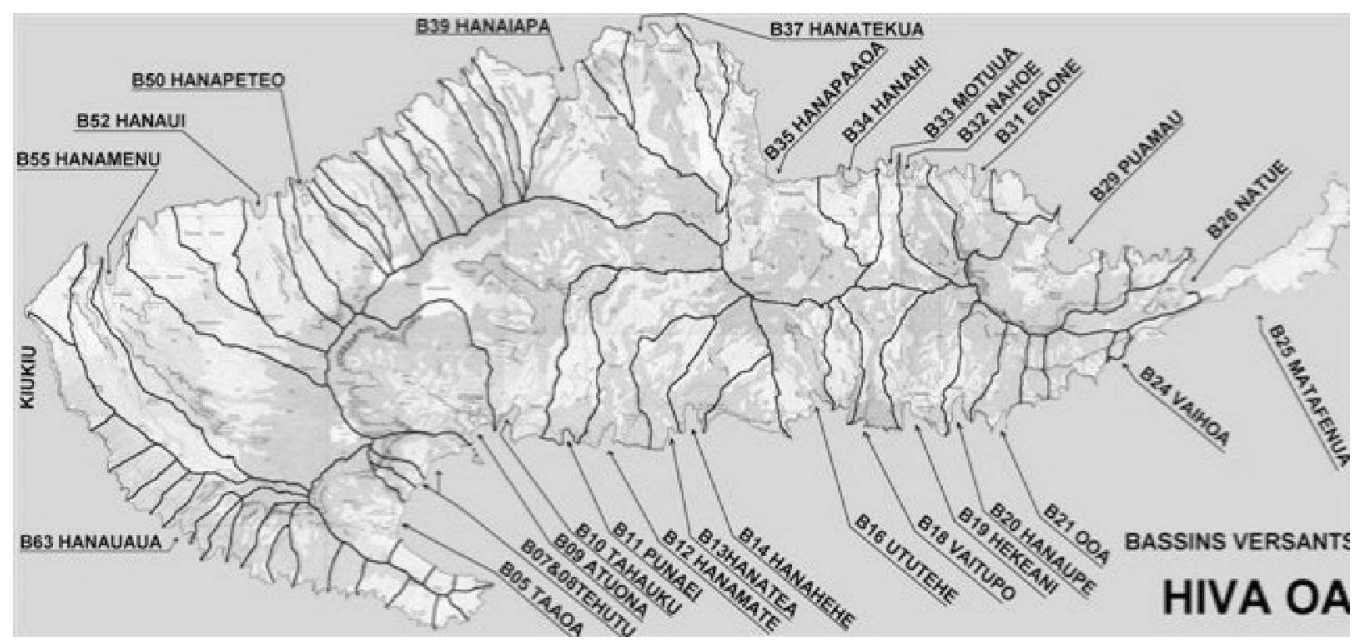

Figure 1. - Carte de l'île découpée en bassins versants

de dessins cotés de leurs objets, la plupart des autres d'un descriptif écrit, d'un schéma explicatif provisoire et de photographies. Certains sites, répertoriés par Ralph Linton ou étudiés par d'autres, n'ont pu être reconnus et situés parce que détruits ou dépourvus de leurs attributs reconnaissables (tiki). Tous les sites répertoriés ont été débarrassés des branches mortes et du petit sous-bois, mais sans toucher aux alluvions qui recouvrent les dallages, sauf devant les dalles sculptées.

\section{Les grands ensembles cérémoniels}

\section{Le tohua, centre de cohésion du groupe}

Un tohua est une place rectangulaire, entourée partiellement ou complètement de constructions, où avaient lieu les cérémonies.

« Il permettait de réunir l'ensemble de la communauté, et bien plus, lors de fêtes commémoratives des funérailles de grands chefs, par exemple. C'est le chef de la tribu qui décidait de sa construction et les spécialistes qui dirigeaient les travaux auxquels la population participait. [...] Le tohua, développement architectural propre aux Marquises, jouait donc un rôle communautaire essentiel au maintien de la cohésion sociale du groupe par le biais des activités qu'il suscitait ou rendait possible. » (Ottino, 1999a : 19)

Certains tohua sont entièrement clos par un alignement de blocs, ou entourés de paepae et de plates-formes dont certaines s'étagent en gradins destinés à recevoir la population et les invités (Upeke B05-01, Hekokua B09-06-01, Makamea
B11-10-03, Faepoto B13-01-03, Hanauaua B6302, probablement Manavaii B18-01); d'autres comprennent des ouvertures entre les structures (Pekia B09-01-01, Faeani B09-20-01, Manavai B12-01, Teotina B14-03-01); d'autres encore sont ouverts sur un ou deux côtés (Matapihi B13-02-01, probablement Tehueto B10-01-02 et Utukua B11-07-01). À Hiva Oa, la place de danse elle-même dépasse rarement $50 \mathrm{~m}$ de long (alors qu'elle peut atteindre $150 \mathrm{~m}$ à Nuku Hiva), ses proportions sont d'habitude d'un tiers de largeur pour deux tiers de longueur. Certains types de structures associées se retrouvent autour de la plupart des tohua: le tuu est une plate-forme sacrée à peu près carrée, entourée de dalles de $k e^{\prime} e t u^{5}$, où avaient lieu les sacrifices. Sur le tohua Upeke (Taaoa, B05-01) et sur celui de Manavai (B12-01), le tuu occupe une extrémité ; sur d'autres, il est inclus dans une des platesformes d'un des grands côtés comme à Teotina dans la vallée de Hanahehe (B14-03), à Hekokua dans celle d'Atuona (B09-06) ou à Manavaii (B18-01). D'autres différences se manifestent dans la couverture de la structure (généralement un pavage de galets), la présence de bas-reliefs ou la profondeur de la fosse.

\section{Makamea, un exemple d'ensemble cérémoniel} dans la vallée de Punaei (B11)

Cet ensemble représente le centre social et religieux de la haute vallée de Punaei (voir Fig. 2). La hiérarchie sociale et religieuse des différents lieux s'harmonise avec les caractéristiques du terrain. Certains espaces ont été nivelés et parfois agrandis en taillant verticalement les

5. Ke'etu: tuf volcanique généralement taillé en dalles utilisées comme parement de façades de paepae ou de terrasses réservés à l'élite. Les $t i k i$ sont bien souvent sculptés dans cette matière. Il existe de nombreuses sortes de $k e$ 'etu : tuf rouge tendre parfois mêlé de scories dures, ke'etu gris et dur, jaune... 


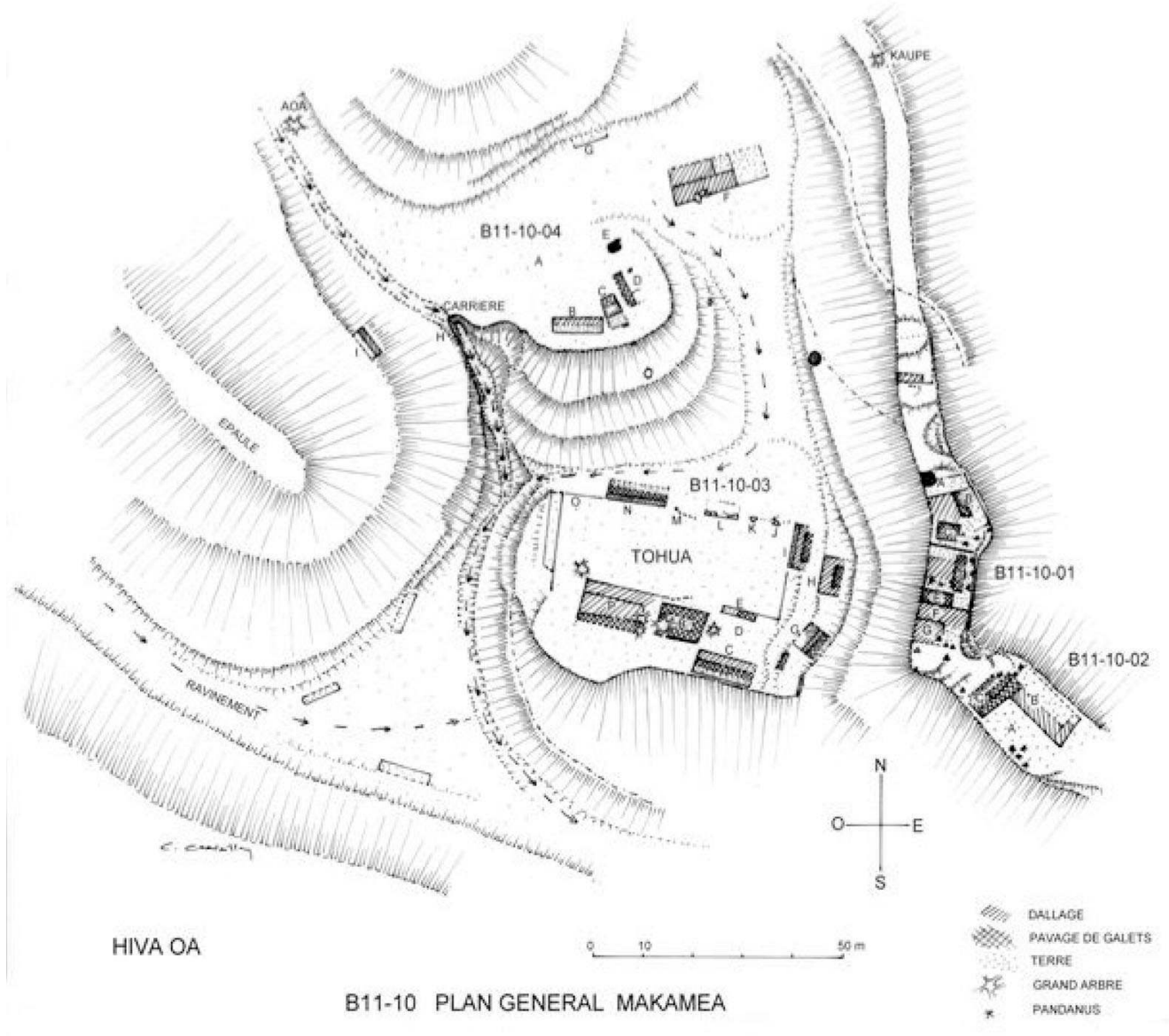

Figure 2. - Plan de l'ensemble cérémoniel de Makamea

pentes. Les voies de communication entre les différents secteurs d'activité sont encore très lisibles ; les zones d'habitations, les enclos, les fosses se répartissent avec une grande cohérence.

Il se compose d'un me'ae de crête (B11-10-01 et -02), d'un tohua (-03), d'un site d'habitation et d'une grande maison (peut-être la maison des guerriers), ainsi qu'une carrière de ke'etu taillée dans le ravin qui borde à l'ouest le site d'habitation (-04). Celui-ci comprend deux paepae séparés par une petite structure bordée de ke'etu surmontée de grandes pierres (pouvant représenter l'espace sacré familial) et une fosse non parementée aux parois verticales. La grande maison siège au dessus du passage permettant de descendre sur le tohua en suivant le ravinement ou de monter sur la crête jusqu'au pied de la partie nord du me'ae par un sentier contournant un ua ma (fosse silo). Quelques plates-formes pouvant correspondre à des soubassements d'habitations sont installées aux abords du site.

$\mathrm{Au}$ Nord, le me'ae est protégé par une plate- forme et les pentes abruptes de la crête ; au Sud, plusieurs paepae gardent le passage.

\section{Le me'ae Makamea}

Le me'ae Makamea couvre toute la largeur de la crête ( 8 à $10 \mathrm{~m})$, ses deux parties sont séparées par un petit sommet couvert de toa (aito, bois de fer Casuarina equisetifolia). La partie nord (B1110-01) de $33 \mathrm{~m}$ de long, étage ses trois grandes terrasses aménagées de plates-formes bordées de dalles de ke'etu à partir d'une fosse rectangulaire aux parois verticales jusqu'à l'alignement traversant la crête au bord du sommet. Une pierre dure, brute, de $122 \mathrm{~cm}$ de haut sur $60 \mathrm{~cm}$ de large est plantée verticalement dans le pavage au milieu de la deuxième terrasse. La face plane de la pierre dressée est orientée vers le Nord dans l'axe du me'ae et coupe perpendiculairement celui du tohua. Plusieurs dalles creusées de cupules sont placées parmi les dalles de couverture des murs; d'autres sont intégrées aux pavages des plates-formes. Des débris d'os longs appa- 


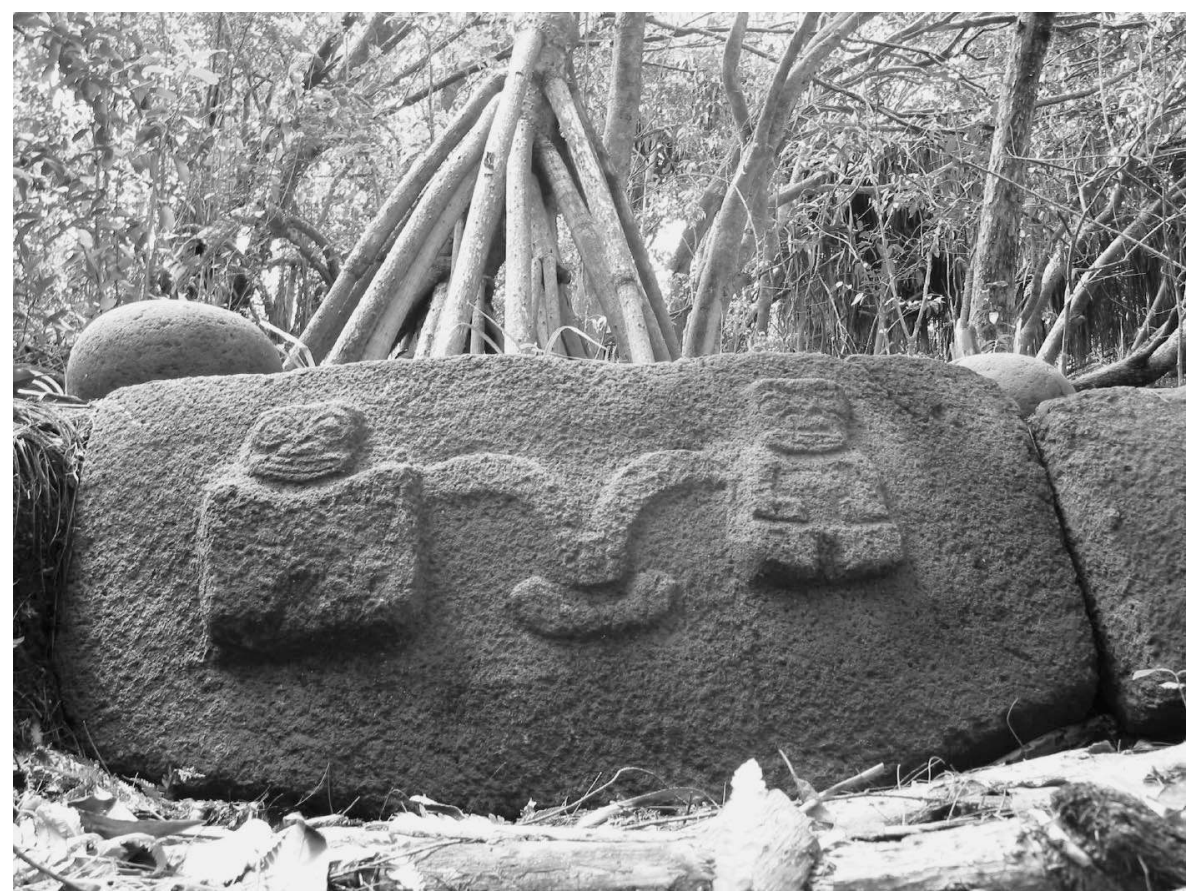

FigURE 3. - Dalle sculptée de deux personnages reliés entre eux par un ka'ake et un poka'a (B10-10-02), Makamea

raissent entre les blocs du mur de soutien de la deuxième terrasse. Ralph Linton a visité et décrit ce site en 1920, visiblement impressionné par la profondeur de la fosse et l'austérité de l'endroit, mais ses guides ont omis de lui montrer la seconde partie. Derrière ce petit sommet et après le changement d'orientation de la crête, la partie sud du me'ae (B11-10-02) consiste en une place rectangulaire de $15 \mathrm{~m}$ de long et de $7 \mathrm{~m}$ de large flanquée d'une grande terrasse à l'Est et fermée au Nord-ouest par une structure à trois niveaux. La façade du deuxième niveau est constituée de dalles de ke'etu placées sur chant dont quatre sont sculptées d'une paire de bas reliefs rectangulaires. Sur la plus belle (voir Fig. 3), deux personnages sont reliés entre eux par un lien plongeant dans une coupe que l'on peut comparer aux motifs de tatouage $k a^{\prime} a k e^{6}$ et poka' $a^{7}$. Les terrasses sont bordées de belles pierres plates parmi lesquelles sont placés un aiguisoir et cinq pierres à cupules.

\section{Le tohua de Makamea (B11-10-03)}

L'espace de danse est long de $47 \mathrm{~m}$ et large de $20 \mathrm{~m}$ en moyenne. C'est un tohua entièrement clos par un alignement plus ou moins dense de gros blocs, entouré de huit structures et ombragé de grands hutu-Barringtonia asiatica (voir Fig. 2).

Le tuu (B) est placé au milieu de la longueur sud, les grandes dalles de bordure sont de ke'etu rouge ou blanc. Le long de la place de danse et sur son côté ouest, six d'entre elles sont sculptées de bas-reliefs (l'un d'eux est anthropomorphe) ; une dalle taillée dans un bloc de corail s'intercale dans l'alignement du côté sud. Sa surface est recouverte de gros galets de plage harmonieusement calibrés : les plus gros sont placés sur les bords extérieurs, les plus petits délimitent l'espace rectangulaire ménagé au centre, plus bas de $16 \mathrm{~cm}$ et apparemment non pavé. Le paepae (C) semble représenter la maison du chef en raison de son emplacement privilégié (à côté du $t u u$ ), ses dimensions, la qualité du dallage du premier niveau, le pavage de galets du second et son ornementation. Il se trouve en retrait de l'alignement de bordure de la place, deux dalles sculptées de bas-reliefs ornent la façade de son second niveau.

L'entrée du tohua s'ouvre sur le petit côté

6. « Le motif [ka'ake] s'apparente aux tracés en équerre et dérive du rectangle, aussi bien que du mouvement de bras des tiki. [...] Il fait partie des symboles de liens et "nœuds magiques" portés par les chefs, les prêtres. [...] Tilésius les mentionne parmi les marques d'appartenance à la table d'un chef, ou celles qui font obligation à la population de nourrir ceux qui sont ainsi tatoués, même en cas de disette. » (Ottino-Garanger 1998 : 112).

7. « Le poka'a : l'idée de quelque chose de circulaire, un peu ovale, de puit, creux [...], de fendre, ouvrir [...], gousse, enveloppe [..., ] ténèbres [...], femme » (Ottino-Garanger $1998: 261)$. 


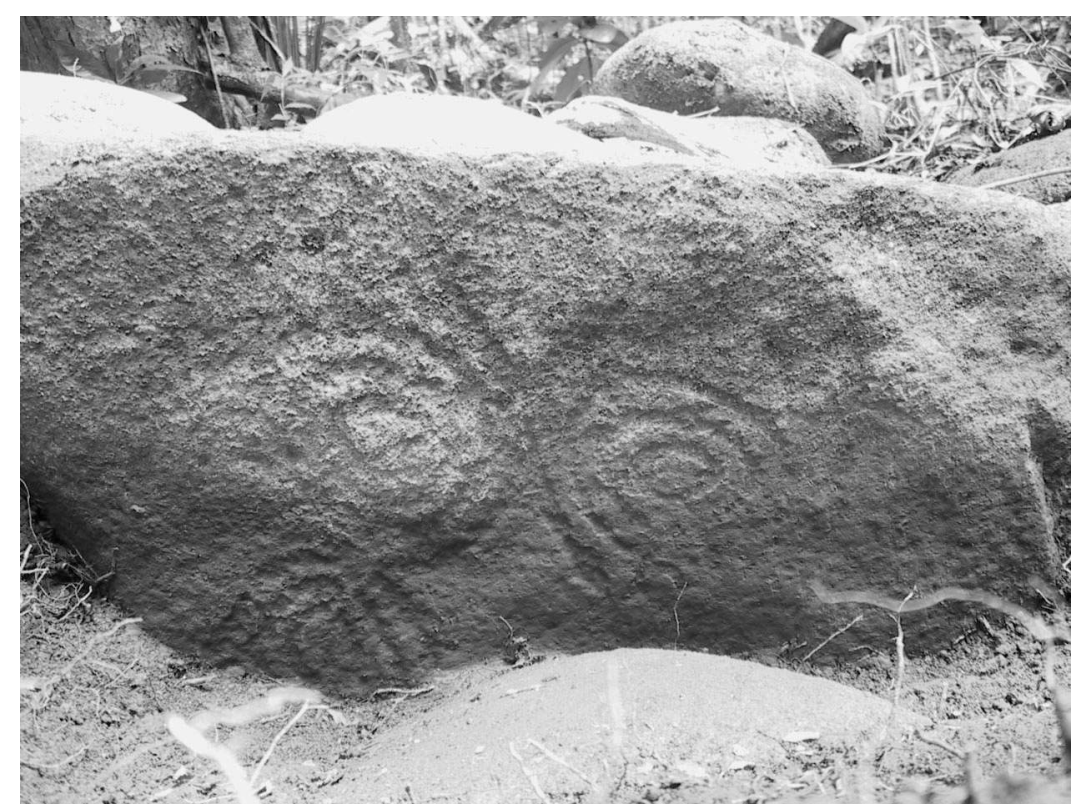

Figure 4. - Dalle (p) gravée de pétroglyphes Mata et Ipu, située à l'extrémité est du tohua de Makamea

ouest le long de la rivière. Sur le côté opposé trois structures s'étagent sur le versant de la crête qui supporte le méae. Un pétroglyphe (voir Fig. 4) figurant trois séries de formes circulaires concentriques $\left(\right.$ mata $^{8}$ et $\left.i p u^{9}\right)$, gravé sur une dalle $(\mathrm{p})$, enrichit la façade de la structure la plus haute $(\mathrm{H})$, vraisemblablement réservée aux prêtres.

Deux séries de plates-formes en gradins $(\mathrm{P})$ et (N) se font face dans la première partie des grands côtés nord et sud. Trois polissoirs aiguisoirs et un galet à cupule se trouvent sur le tuu, deux pierres à cupules et un polissoir sont placées sur les structures du petit côté est du tohua, trois autres sur le côté nord, une autre sur la structure en gradins sud.

Deux « tables » de pierre matérialisent le bord nord-est du tohua. Elles sont composées d'un grand plateau de pierre d'environ $1 \mathrm{~m}$ de long supporté par trois grosses pierres ou davantage. Parfois, ces «tables» placées en bordure de place de cérémonies sont intégrées au mur de clôture comme celles des tohua de Utukua (B1107), Atuona (B09-06-01), Tahauku (B10-01-04) et Manavai (B12-01).

\section{La pierre, support de l'expression symbolique}

\section{Les pétroglyphes}

À Hanaiapa, un rocher (voir Fig. 5) long de 6 m, gravé de pétroglyphes et nommé Hakaiki $(\mathrm{B} 39-01 \mathrm{H})$, présente sur la presque totalité de sa surface des dessins plus ou moins imbriqués parmi lesquels on reconnaît des motifs de tatouages (ipu, eipuoto, mata, poka'a) et une quinzaine de enata (représentations humaines) reliés entre eux, ou associés à d'autres signes. De l'autre côté de la rivière se trouve le me'ae associé au tohua Koiomai (B39-02).

Un autre rocher gravé est situé à proximité du me'ae Tehueto de Tahauku (B10-01) et un autre encore à Ooa (B21-02). À Hanapaaoa, une grande dalle carrée couverte de pétroglyphes sur une face et une tranche a été placée au bord du dallage d'une imposante terrasse (B35-03). Une photographie de Ralph Linton (1920) la montre en situation sur le mur, sa tranche gravée apparaissant grâce à l'absence de la dalle voisine. Depuis, la dalle gravée est, elle aussi, tombée du mur, révélant sa face cachée couverte de pétroglyphes. S'agit-t-il d'une réutilisation de la dalle

8. Ce mot désigne l'extérieur d'une chose, une face, l'œil, le regard.

9. Le ipu est un motif en forme d'anneaux, souvent au nombre de trois ; il représente un élément circulaire, une représentation de l'univers. Le chef régent Paiore explique à Eugène Caillot : "L'univers était comme un œuf... Il finit par crever et donna naissance à trois plates-formes superposées, celle du bas supportant les deux autres. Sur la plate-forme inférieure demeurèrent Te Tuтu (fondation) et Te Papa (roc stratifié) qui créèrent l'homme, les animaux, les plantes [...] » (Ottino 1998 : 210-211). On retrouve ce modèle dans l'organisation des plates-formes des me'ae. Le motif ipu est très largement utilisé dans l'art du tatouage, la sculpture sur bois et sur pierre, les pétroglyphes. 


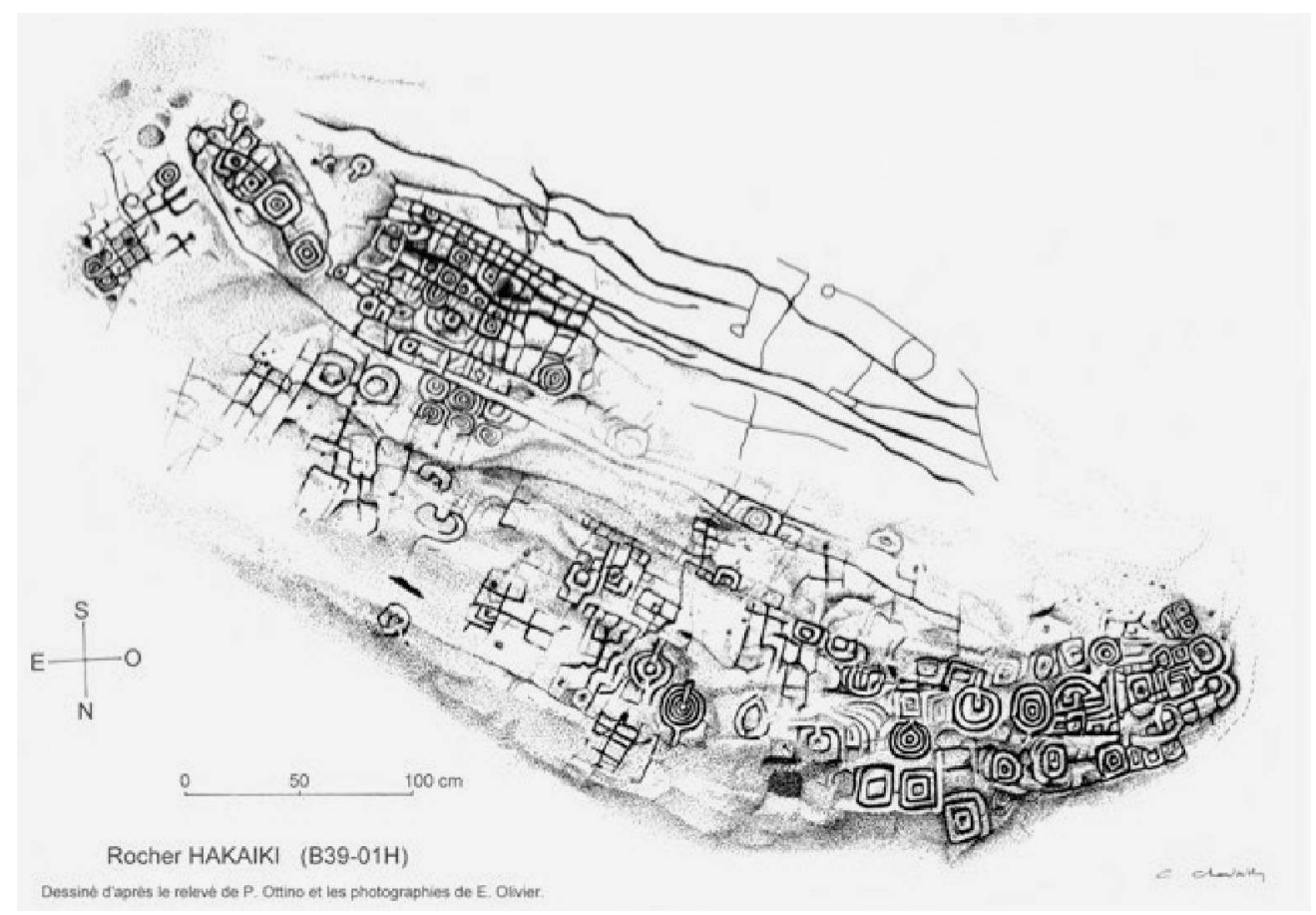

Figure 5. - Rocher Hakaiki B39-01H

(Dessin réalisé d'après le relevé de Pierre Ottino et les photographies d'Éric Olivier)

ou ces dessins étaient-ils destinés à rester cachés ? La réutilisation d'une pierre de cette sorte pouvait correspondre à l'appropriation de son $m a n a^{10}$ ou de celui de son lieu d'origine. Sur de nombreux sites, la partie inférieure des basreliefs de certaines dalles de ke'etu se trouve dissimulée sous le niveau du dallage de leur plate-forme, appuyant l'hypothèse que ces objets n'avaient pas pour seule fonction l'ornementation de l'édifice, mais participaient à son mana en s'intégrant à son infrastructure. L'exemple le plus spectaculaire est le me'ae du tohua de Manavai. Son dallage masque totalement la bouche des deux grosses têtes de tiki ornant sa façade et il ne semble pas que les sculptures se soient enfoncées après leur mise en place.

Dans la plupart des cas, les pierres gravées de 1 à 6 motifs plus ou moins reliés entre eux, sont placées sur chant parmi les dalles de façade de certaines terrasses ou sont comprises dans un pavage (dans ce cas, il s'agit parfois d'un bloc à la surface arrondie ou d'un galet). Les formes concentriques (mata, ipu, poka'a), (dans certains cas par séries de trois ou davantage) et les enata (humains) sont les motifs les plus couramment rencontrés. Plus rarement un etua (divinité), le visage d'un tiki ou un animal (un cachalot [B1210] et un gecko [B31-01]). Ces pétroglyphes paraissent, pour la plupart d'entre eux, réalisés par piquetage.

\section{Les dalles de ke'etu sculptées}

Les dalles de ke'etu sculptées en bas-relief sont habituellement placées au centre de la façade d'un tuu, de la façade du second niveau d'un paepae de dignitaire ou de celle d'une des platesformes supérieures d'un me'ae. Très nombreuses, elles sont ornées pour la plupart de figures anthropomorphes (tiki, enata) plus ou moins abouties (du rectangle en relief au tiki finement sculpté), moins souvent zoomorphes ou composites (lézard, homme-lézard, lézard-poisson, poisson, tortue), quelques-unes évoquent des signes de tatouage (ka'ake, poka'a).

D'autres dalles de ke'etu présentent une surface gravées, comme avec une gouge et un percuteur, de motifs géométriques. Les enlèvements sont suffisamment nets pour que l'on puisse se représenter les petits coups rythmés qui ont sculpté ces lignes. Ces dalles sont placées sur des taha tupapau (petite structure haute destinée au traitement des cadavres et souvent réceptacles d'ossements) ou sur des me'ae dans les vallées de Atuona (B09-17), de Punaei (B11-07) et de Hanatea (B13-01). Les lignes sculptées forment

10. Puissance, pouvoir, énergie, caractère sacré émanant d'une divinité, d'un ancêtre, d'une personne, d'une chose et pouvant se transmettre de génération en génération. 


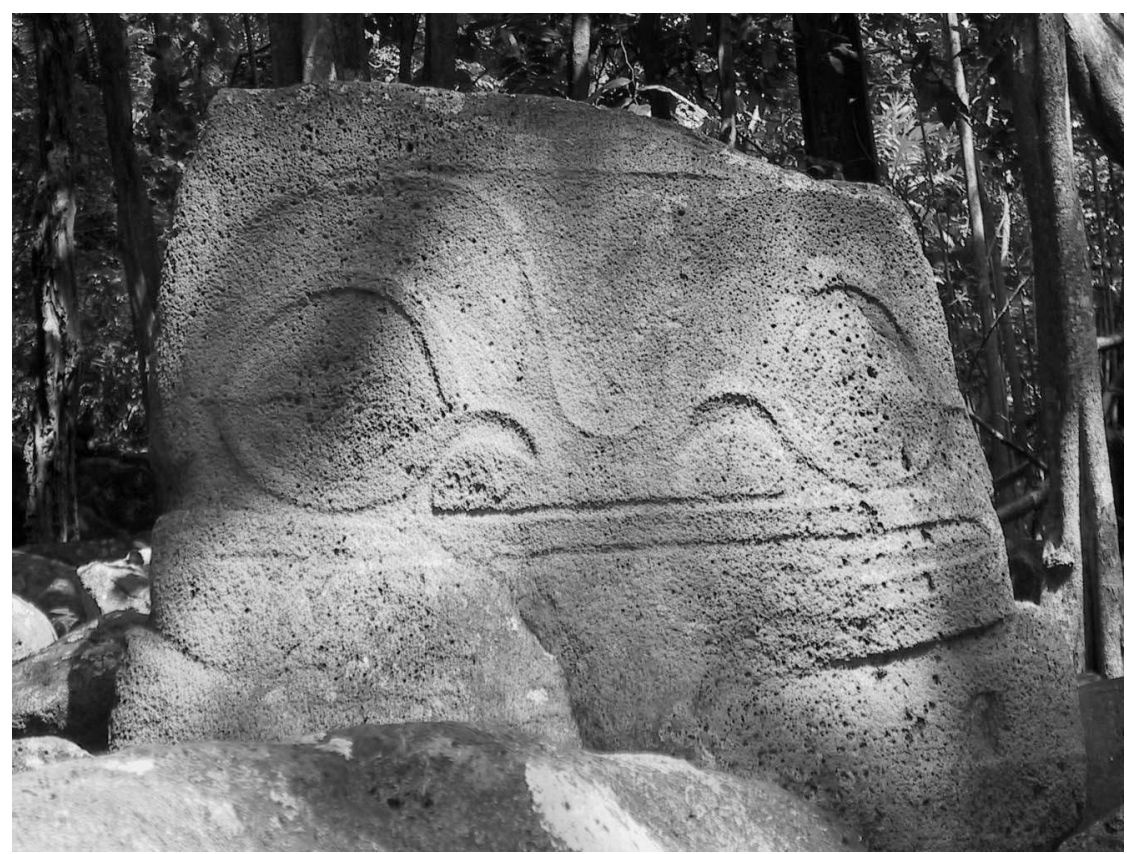

FIGURE 6.- Tiki. Upeke (Taaoa)

des bandeaux de traits parallèles horizontaux ou verticaux, s'organisant en zigzags, en V emboîtés ou en arceaux. Ces décorations rappellent le travail de laçage et les décorations gravées des pou, poteaux principaux des maisons. Les dalles sculptées de bandes horizontales ou verticales semblent plus couramment utilisées, le plus souvent sur des me'ae. Ces lignes strient parfois la surface de fond des dalles sculptées de basreliefs. La grande majorité de ces dalles se trouvent dans les vallées de la partie sud de l’île.

\section{Les tiki}

Les tiki sculptés en statues comportent généralement un prolongement sous leur base de façon à les planter solidement dans le pavage d'une terrasse. Ceux d'Atuona ont tous été enlevés des sites au cours $\mathrm{du} \mathrm{Xx}^{\mathrm{e}}$ siècle. Deux exemplaires se trouvent au musée de Tahiti et des îles, identifiables grâce aux photographies de Ralph Linton. Le corps renversé et décapité d'un grand tiki de ke'etu rouge est resté sur le me'ae Ahuahu.

À Puamau en revanche, de nombreux tiki demeurent en place. Le grand me'ae Iipona étudié et réhabilité par P. et M.N. Ottino (1991) en compte cinq dont le grand Takaii (hauteur : 2,60 m) et l'étrange tiki couché Makii Taua Pepe. Un autre tiki de ke'etu rouge $(1,20 \mathrm{~m})$ se trouve encore sur le petit me'ae Haapetai. A Hanapaaoa, le tiki Moeone domine toujours la plate- forme supérieure du me'ae funéraire de crête Tapuohe (B35-01).

Le tiki de Taaoa (B05-01), exceptionnellement sculpté sur un grand bloc de pierre dure, haut de $1,30 \mathrm{~m}$ et large de $1 \mathrm{~m}$, garde le labyrinthe de plates-formes du me'ae qui surplombe le tohua Upeke (voir Fig.6).

Les tiki en forme de stèle sont généralement plus petits et plus stylisés. C'est le cas du tiki souriant de Utukua dans la vallée de Punaei (B11-07), ainsi que deux autres à Hanaiapa (B39-04) et à Atuona (B09-22).

Certains tiki sont sculptés sur des blocs de ke'etu insérés dans une façade de façon à ce que le personnage paraisse se détacher de l'alignement des dalles, tout en étant solidement ancré dans la structure. À Puamau, cinq d'entre eux ornent la façade du paepae Poevau, cinq se trouvent sur le site du tohua Pehekua, deux gisent en bas de leur structure : tiki Tefififi (B29-04-01), les autres ont été déplacés. À Atuona, le petit me'ae Aaha a conservé ses trois tiki.

Des têtes isolées sont parfois insérées dans les murs de façade de me'ae. La tête en ke'etu rouge d'un me'ae de la vallée d'Atuona (B09-07) et celle de ke'etu blanc du me'ae de Tehueto (B1001) sont encore en place.

Certains tiki sont ornés de tatouages sculptés en relief sur le visage : ceux de Pehekua, celui de Haapetai et celui de la mission de Puamau ont les joues tatouées; le tiki de Punaei porte de très fins tatouages labiaux. Takaii de Iipona (Puamau) et Moeone de Hanapaaoa ont le corps 
gravé de lignes parallèles. Horizontales sur les jambes de Moeone, elles se brisent dans son dos en chevrons dont les pointes supérieures dessinent la colonne vertébrale, des cercles concentriques ornent ses fesses. Sur celui de Takaii, les yeux montrent encore les traces de lignes verticales, mais la surface du colosse de ke'etu rouge est trop dégradée pour être encore lisible. Son moulage, réalisé en 1956 pour le musée Kon Tiki en Norvège, témoigne de lignes gravées en zigzag sur sa jambe droite et sur le pectoral gauche.

\section{Les peintures rupestres}

Lors de leur mission de 1985, Sidsel Millerstrom et Edmundo Edwards ont identifiés six abris rocheux contenant des peintures rupestres sur le flanc est de la vallée de Eiaone. D'après les auteurs, les dessins peints à l'ocre représentent des humains et des animaux : chiens, oiseaux, poissons, langouste et peut-être des êtres hybrides (homme oiseau). Les promeneurs ne sont pas les bienvenus dans cette vallée, nous ne l'avons pas encore visitée.

\section{Les pierres à cupules et aiguisoirs}

Les pierres à cupules du tohua de Utukua (B11-07) sont réparties parmi les dalles qui recouvrent les plates-formes installées le long de son grand côté ouest. Un bel aiguisoir se trouve dans l'espace réservé du me'ae. Dans l'enceinte du site Upeke à Taaoa, on compte soixante et une pierres de ce type (le mur de clôture du tohua comprend plus de pierres à cupules que de polissoirs, le me'ae associé au tohua et le me'ae supérieur comportent davantage de polissoirs et d'aiguisoirs que de cupules.)

Certaines grandes pierres comprennent jusqu'à treize cupules, d'autres associent les cupules et les rainures d'aiguisage; certaines, plus rares, comprennent un grand nombre de cupules et une excavation rectangulaire à fond plat profonde de $4 \mathrm{~cm}$ environ (Puamau Iipona B29-01 et B29-04-02). Trois pierres portent à la fois une cupule et un ou plusieurs pétroglyphes, ces pierres bordent des structures intégrées à des ensembles complexes de type me'ae.

Les aiguisoirs comportent parfois une ou deux entailles, d'autres en associent plusieurs séries sur le même plan ou sur plusieurs côtés d'un galet allongé, d'autres encore sont de grands rochers généralement situés aux abords d'un cours d'eau, dont la totalité de la surface utilisable est divisée en alvéoles polies et striées (B05 Taaoa [2], B09 Atuona, B13 Matapihi, B21 Ooa, B39 Hanaiapa).
La plupart de ces pierres se trouvent à leur place sur les sites, parce que trop lourdes à transporter ou trop éloignées des pistes (il est rare de rencontrer un objet travaillé de petite dimension). Le diamètre des cupules varie généralement entre $6 \mathrm{~cm}$ et $15 \mathrm{~cm}$ avec une profondeur de $1,5 \mathrm{~cm}$ à $6,5 \mathrm{~cm}$, exceptionnellement plus de 20 $\mathrm{cm}$ avec une profondeur de plus de $8 \mathrm{~cm}$.

Certaines pierres, dont les cupules présentent une trace de travail leur donnant une forme ovoïde, peuvent révéler la position qu'adoptait la personne qui l'utilisait. D'autres sont, à n'en pas douter, des réutilisations : intégrées à certaines façades de paepae ou de plates-formes et présentant leur face travaillée verticalement, elles étaient à la fois respectées et inutilisables.

\section{Les me'ae}

\section{Les me'ae et lieux de réunion}

Le plus souvent, les me'ae ou les lieux de réunion, observés à Hiva Oa, sont constitués d'au moins trois niveaux étagés, chaque niveau pouvant comprendre plusieurs terrasses disposées en $\mathrm{L}$ ou en U. Ces niveaux successifs s'échelonnent parfois en escalier, d'une façon pyramidale ou suivant un plan asymétrique dicté par le relief et l'utilisation du site. Le niveau supérieur contient souvent une structure centrale entourée de dalles ou de blocs de ke'etu et recouverte de galets de plage. Les dalles sculptées de bas-reliefs se trouvent généralement parmi celles qui ornent la façade de cette structure ou de celle d'une des plates-formes des côtés. Des ossements (le plus souvent des os longs brisés, rarement un ou plusieurs crânes), des dents humaines et porcines, sont habituellement placés entre les pierres des murs de soutien du me'ae ou d'une de ses structures, plus rarement éparpillés sur les dallages, comme sur les me'ae funéraires de Hanapaaoa (B35-01) et Hanaiapa (B39-04). Des morceaux de corail (blocs taillés ou rameaux) sont parfois enfouis dans les pavages des structures. Particulièrement dans la vallée de Punaei, le corail brut est taillé en dalles placées parmi celles, de ke'etu, qui composent la façade d'une structure ou d'une plate-forme.

Les ensembles complexes de structures, présentant ces caractéristiques architecturales mais ne recelant apparemment pas d'ossement et ne jouissant pas d'une situation exceptionnelle, représentent une grande partie des me'ae rencontrés. Nous avons repris la classification généralement admise. En ce qui concerne les sites inédits, cette attribution a peut-être été donnée à 
tort dans certains cas. Les lieux de réunion peuvent-ils être confondus avec les me'ae? Le tapu frappait pour la plupart des habitants de nombreux lieux, qu'ils soient réservés aux guerriers, aux chefs et aux prêtres ou seulement aux grands prêtres. Les nuances de leur hiérarchie et de leurs utilisations ne sont pas toujours très claires et demandent plus d'informations.

En dehors des me'ae construits sur les crêtes et les épaulements, d'autres aussi nombreux sont installés sur les pentes à proximité des cours d'eau ou proches des sommets. Le grand site de Faepoto (B13-01) s'étend entre deux rivières en haute vallée de Hanatea. Un grand tohua et plusieurs maisons de dignitaires ornées de dalles sculptées en occupent la partie inférieure, une dizaine de petits ensembles de structures aux plates-formes disposées en U ou en L, de terrasses disposées en escalier, de taha tupapau s'égrènent sur la pente jusqu'à la crête.

\section{Faepoto, le me'ae du lézard poisson (B13-01-09)}

Ce me'ae est construit en hauteur, à une vingtaine de mètres au dessus d'une série de terrasses en escaliers comprenant plusieurs bas-reliefs représentant des tiki et d'un ua ma de $11 \mathrm{~m}$ de diamètre et 2,60 $\mathrm{m}$ de profondeur. Le me'ae est composé de trois plates-formes étagées (Voir Fig.7). Sur la plate-forme inférieure pousse un grand aoa (banian), les façades des terrasses des côtés sont composées de dalles de ke'etu ainsi que la structure supérieure carrée (A) couverte de galets de plage soigneusement rangés. La dalle (a), de ke'etu rouge, située au milieu de sa façade sud-ouest est sculptée de deux motifs en bas-relief. Celui de gauche est un petit personnage en mouvement (danseur ?) l'autre est trop détruit pour être interprétable. La dalle (b) de ke'etu gris (voir Fig.8), qui orne la façade sud-est, était enterrée aux deux tiers sous les colluvions. Elle représente un être composé de la partie arrière d'un corps de lézard et de la partie avant d'un poisson, peut-être d'un requin comme l'extrémité avant se termiant abruptement à angle droit par une série d'encoches en dents de peigne et les yeux placés sur les côtés le laisseraient supposer. L'animal se dirige vers un motif composé de rectangles irréguliers qui pourrait représenter un mur ou un paepae.

Les autres ensembles de structures de ce grand site de Faepoto renferment d'autres dalles sculptées de bas-reliefs figurant pour la plupart des tiki, des enata ou des etua. Le seul animal représenté est un lézard vertical ornant la façade du niveau supérieur d'un grand paepae associé au tohua. Dix grandes dalles de ke'etu, décorées de motifs de laçage gravés comme à l'aide d'une gouge, sont réparties sur deux taha tupapau sis en amont du tohua.

Le site naturel bénéficie de pentes douces et fertiles au pied des pentes abruptes de la grande dorsale de l'île, tout au fond de la vallée de Hanatea. La beauté de ses espaces, ses grands arbres et l'étrangeté des rochers comme échoués ou émergeants du sol (une roche grise comprenant des bulles et des rainures naturelles) ont été respectés et employés à organiser un ensemble cérémoniel et religieux d'une grande majesté.

\section{Atuona, le me'ae Pouau (B09-03)}

C'est, d'après Ralph Linton, le me'ae plus important de la vallée d'Atuona. Construit sur le plateau situé à environ $500 \mathrm{~m}$ d'altitude, au-dessus de l'épaule qui sépare le fond de la vallée d'Atuona de la vallée adjacente d'Atikua, il est aussi l'un des plus vastes de l'île. Ses huit ensembles de structures, qui paraissent reliées par des pavages, couvrent le plateau sur plus de $250 \mathrm{~m}$ dans l'axe de la crête, sur environ $150 \mathrm{~m}$ de large. Les tiki de ke'etu rouge décrits et photographiés par Ralph Linton ont été retirés du site, le plus grand se trouve au musée de Tahiti et des îles (numéro d'inventaire : 426).

\section{Eiaone, me'ae Taaovea (B31-01)}

La crête rocheuse surmontée de pitons qui sépare le fond de la vallée Eiaone de celle de Puamau illustre d'une façon spectaculaire l'idée de coopération de l'homme avec la nature.

La paroi du côté de la vallée de Eiaone est presque verticale et la pente sud-ouest du côté de Puamau très abrupte. L'arête de la crête est étroite (entre $1 \mathrm{~m}$ et $5 \mathrm{~m}$ ) et constituée de bosses de ke'etu rouge sombre, granuleux, alvéolé et dur, alternant avec une sorte de dorsale de pierre gris clair, dure, se fragmentant à angle droit et imitant les constructions humaines.

Sur une distance d'environ $400 \mathrm{~m}$, quatre emplacements se répartissent dix panneaux gravés de pétroglyphes (pour la plupart répertoriés par Sidsel Millerstrom en 1985) sur plusieurs rochers aménagés (B31-01-02, -03 et -05). Les aménagements consistent en perforations des arêtes des panneaux de pierre (parfois trois par côté). Les perforations sont souvent ouvertes, c'est-à-dire que l'arête horizontale ou verticale a cédé entre les deux trous. Certaines sont encore intactes, les traces de piquetage qui marquent les parois du trou évasé à l'extérieur et se rétrécissant au centre sont bien visibles. Ces perforations sont pour la plupart circulaires, quelques unes 


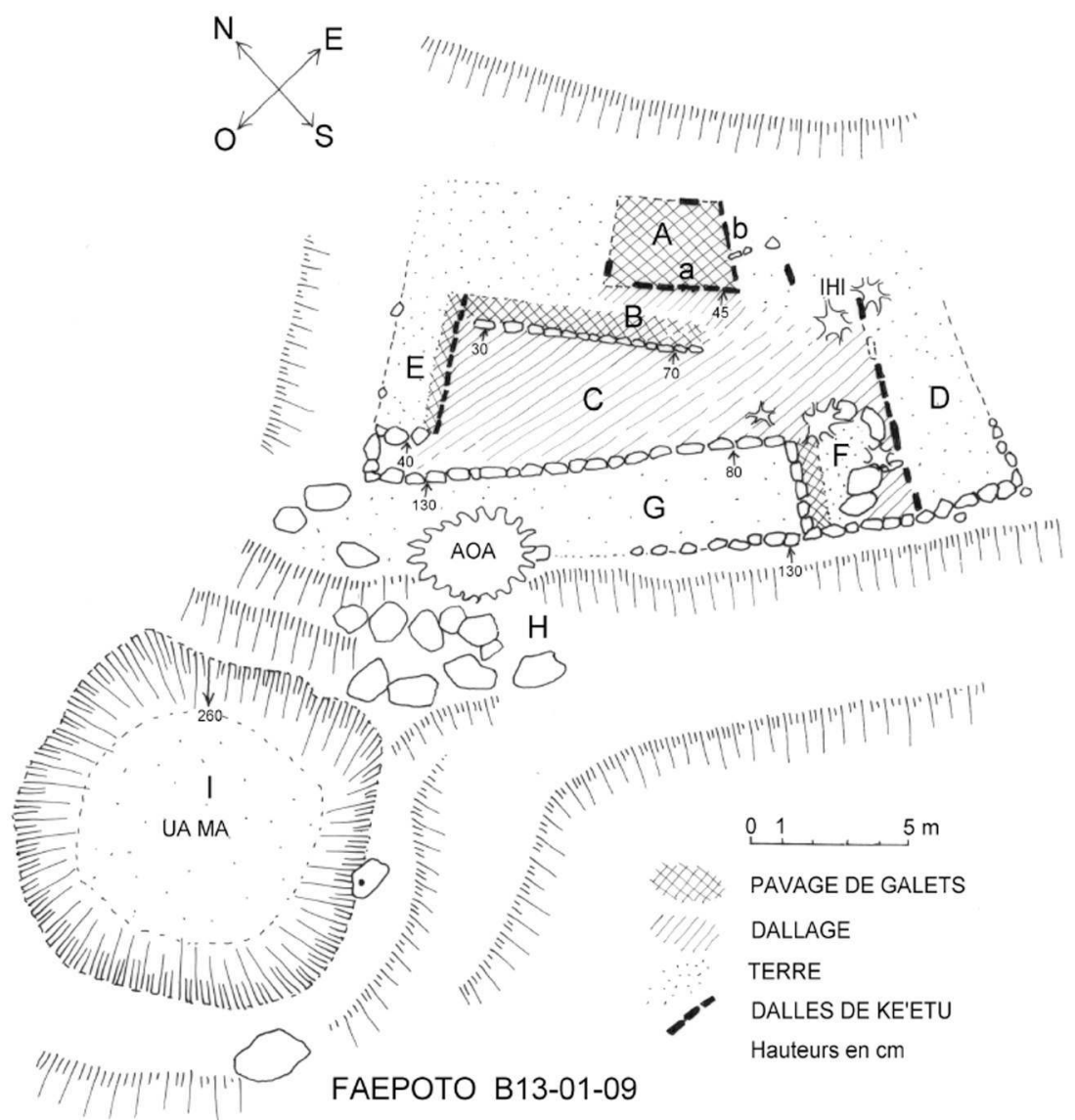

Figure 7. - Plan du méae (B13-01-09)

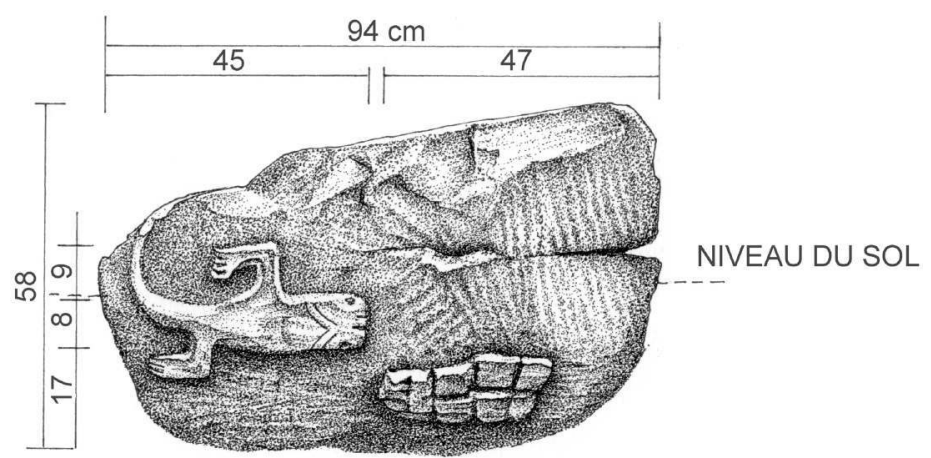

Figure 8. - Dessin de la dalle (B13-01-09b) Faepoto

sont rectangulaires et ressemblent à des poignées (voir Fig.9). Trop irrégulières par la taille et la situation, trop fragiles pour servir à implanter une armature de bois, elles demeurent un cas unique et une énigme dans l'aménagement des rochers de Hiva Oa et peut-être de Polynésie.

Certaines surfaces horizontales de ces rochers ont été creusées par piquetage de plats rectangulaires et d'écoulements. Deux pans de rochers inclinés sont creusés de marches. Entre deux ensembles de rochers travaillés, deux platesformes (B31-01-04) sont installées sur l'arête rocheuse qu'on a complétée par un mur de façade et un dallage (les pierres utilisées paraissent provenir du site). Ni galet, ni dalle de ke'etu, ni ossement n'ont, apparemment, été apportés de l'extérieur.

Le lieu est aérien, vertigineux, remarquable par l'extrême sobriété de ses constructions et la richesse de ses représentations symboliques. Il était probablement réservé à une élite pour des rituels particuliers (l'espace ne permettant pas une assistance nombreuse). 


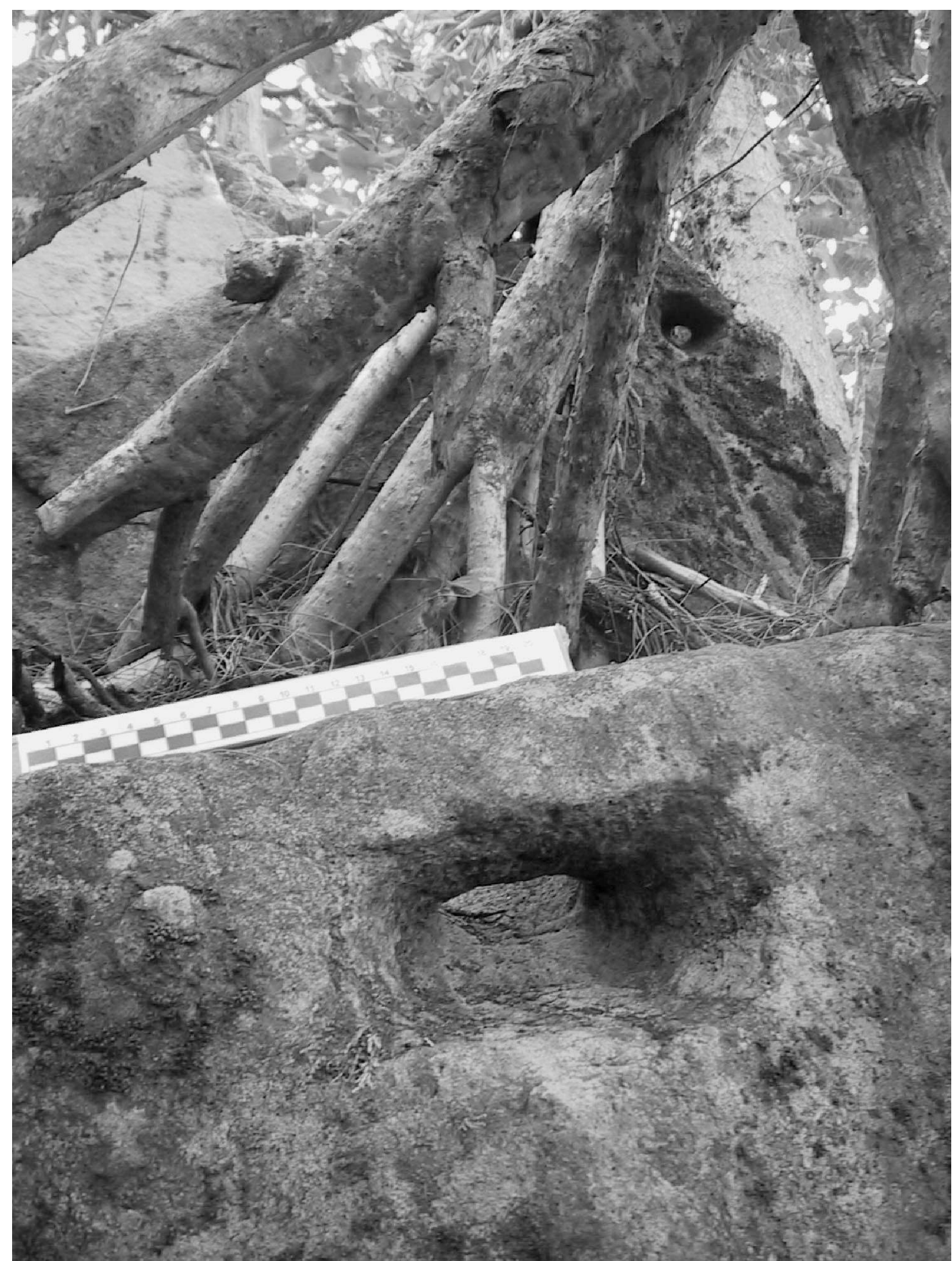

Figure 9. - Me'ae de Taaovea, rocher (B31-01-03).

Au premier plan, perforation rectangulaire ; au deuxième plan à droite, perforation circulaire ; au troisième plan à gauche, pétroglyphe enata.

\section{Conclusion}

Les vestiges des constructions et des aménagements de l'ancienne civilisation témoignent d'une gestion soignée des espaces et des ressources de chaque vallée. Les espèces végétales qui ombrageaient les ensembles cérémoniels : hutu (Barringtonia asiatica), tamanu (Calophyllum inophyllum), maii (Terminalia catappa, badamiers), puatea (Pisonia grandis), ihi (Inocarpus edulis, châtaigner tropical), toa (Casuarina equi- setifolia, arbre de fer), aoa (Ficus sp., banians) sont bien souvent encore présentes sur les lieux et quelques exemplaires des végétaux entretenus dans les jardins subsistent aux alentours des ruines : auti (Cordyline fructifosa), noni (Morinda citrifolia), plans de taros, mei (arbres à pain), ama (Aleurites moluccana, bancouliers).

Les nombreuses pièces encore en place (statues, dalles sculptées, pétroglyphes, pierres à cupules ou aiguisoirs) témoignent de la richesse de l'ornementation et de la sensibilité artistique 
de l'expression symbolique de l'ancienne culture marquisienne. L'étendue, la diversité, la cohérence et la bonne conservation des ensembles de structures qui les abritent appellent à établir un inventaire non seulement des sculptures, mais aussi de leur contexte archéologique. Le recensement des traces d'aménagements comme les fosses à $m a$ (fosses silos), les terrasses de cultures et enclos, les chemins empierrés, rivières canalisées, fosses pièges et lieux de refuge qui subsistent sur un terrain peu exploité, doit compléter cet inventaire. Ces indices et ces structures porteurs de témoignages précieux pour les tentatives d'interprétation vont disparaître à plus ou moins brève échéance : il est donc urgent de les répertorier.

Protéger les sites majeurs, les étudier et dater ceux qui rassemblent le plus d'informations permettrait d'apporter une dimension chronologique essentielle à la compréhension de ces sociétés. Enfin, la réhabilitation et l'entretien de certains sites, en comptant ceux qui sont déjà visités par les touristes et les écoles, participeraient à la reconnaissance et à la conservation du patrimoine marquisien.

\section{BIBLIOGRAPHIE}

Bellwood Peter S, 1972. A settlement pattern survey, Hanatekua Valley, Hiva Oa, Marquesas Island..., Honolulu, Pacific Anthropological records17, Department of Anthropology, Bernice P. Bishop Museum.

Chavaillon Catherine, 2002. Makamea, un ancien centre de vie social et religieux dans la haute vallée de Punaei, Bilan de la recherche archéologique en Polynésie française 2001-2002, ministère de la Culture de Polynésie française, Service de la Culture et du Patrimoine, pp. 69-74.

Clayssen, 1922. Archéologie des îles Marquises, liste de quelques me'ae de l'île de Hiva Oa, Bulletin de la Société des Etudes Océaniennes, n ${ }^{\circ}$ 6, pp. 6-10, réédité en 1989, BSEO 248, pp. 95-99.

CoIfFIER Christian (éd.), 2001. Le voyage de La Korrigane dans les mers du sud, catalogue de l'exposition présentée au musée de l'Homme du 5 décembre 2001 au 3 juin 2002, Hazan-Paris, musée de l'Homme-MNHN.

ConTE Éric, 1995. Dater la colonisation humaine des Marquises : problème et perspectives. Bulletin de la Société des études océaniennes 268, XXIII, 5, pp. 3243.

-, 2000. L'archéologie en Polynésie française, esquisse d'un bilan critique, Papeete, Au Vent des îles.
Delmas Siméon, 1927. La Religion ou le Paganisme des Marquisiens, d'après les notes des anciens missionnaires, Braisne-le-Comte (Belgique)-Paris, Maison mère des Sacrés-Cœurs de Picpus, G. Beauchesne.

Dordillon René I. (Mgr.), 1904. Grammaire et dictionnaire de la langue des îles Marquises, Paris, impr. Belin Frères (rééd. 1999, Papeete, Société des études océaniennes).

EDWARDS Edmundo, 1985. Éléments de description du tohua Pehe Kua, vallée de Puamau, Hiva Oa, Papeete, département Archéologie, centre polynésien des sciences humaines, Te Anavaharau, ms, $21 \mathrm{p}$.

Linton Ralph, 1925. Archaeology of the Marquesas Islands, Honolulu, Bernice P. Bishop Museum, bulletin 23, Bayard Dominick expedition, publication number 10 (rééd. 1971, New York, Kraus Reprint $\mathrm{CO})$.

Millerstrom Sidsel et Claudio Cristino, 1985. Rock Art Project in Marquesas, rapport de mission, Papeete, département Archéologie, centre polynésien des sciences humaines, Te Anavaharau, ms, $442 \mathrm{p}$.

Millerstrom Sidsel, 1985a. Rock Art Project in the Marquesas Islands, a preliminary report, La Pintura, American Rouck Art Research Association Newsletter $12 \mathrm{~h} 7$.

-, 1985b. Up-date on Marquesan rock art, La Pintura, American Rouck Art Research Association Newsletter $12 \mathrm{~h}$ 5-6.

Millerstrom Sidsel et Edmundo Edwards, 1995. Peintures rupestres de la vallée de Eiaone à Hiva Oa, Bulletin de la Société des études océaniennes 267. XXIII, 5, pp. 5-17.

Navarro Maeva, Claudio Cristino, Patricia Vargas et Jean-Luc RIEU, 1986. Document de terrain, Papeete, département Archéologie, centre polynésien des sciences humaines, Te Anavaharau, ms, 4 p.

OLLIER François, 1970. Inventaire des vestiges, monuments et sites d'intérêts archéologiques, historiques et naturels des îles Marquises, octobre-décembre 1970, Papeete, ms, 70 p.

Ottino Pierre et Marie-Noëlle De Berg, 1991. Hiva Oa, images d'une mémoire océanienne, Papeete, département Archéologie, centre polynésien des sciences humaines, Te Anavaharau.

-, 1993. Archéologie et Festival des Arts, le cas de Iipona à Puamau, Hiva Oa, Bulletin de la Société des études océaniennes 256-257, XXI, 7-8, pp. 77-101.

-, 1998 a. Le Tatouage aux Îles Marquises, Te patu tiki, Papeete, Jean-Pierre Fourcade et Christian Gleizal éditeurs, coordination à Singapour, éditions Didier Millet.

-, 1998 b. Carnet de terrain ; le tohua Tahakia à Hatiheu, Nuku Hiva, îles Marquises, Bulletin de la Société des études océaniennes 276, pp. 52-65.

-, 1999 a. L'habitat ancien aux îles Marquises, Revue Horizon Magazine 333, Papeete, pp. 18-35. 
-, 1999 b. Fenua enata : la Terre des Hommes, in Eve Sivadjian, Les îles Marquises, archipel de mémoire, Paris, éditions Autrement, pp. 116-143.

-, 2001. Habitat ancien et protection du patrimoine à Hatiheu : une vallée marquisienne. Journal de la Société des Océanistes 113, pp. 201-214.

-, 2004. Les tikis témoins des origines, Sciences au Sud, journal de l'IRD 27, p. 7.

Peltier Francis Moeava, 1973. Structures préhistoriques d'une vallée des Marquises Hanaiapa, Hiva Oa, Bulletin de la Société des études océaniennes 183 , XV, pp. 272-306.

PÉTARD Paul, 1986. Plantes utiles de Polynésie, Raau Tahiti, édition revue, augmentée et illustrée, Papeete, éditions Haere Po No Tahiti.

Rollin Louis, 1974 (1 $1^{\mathrm{e}}$ éd. 1929). Mours et coutumes des anciens Maoris des îles Marquises Papeete, Stepolde.

Sinoto Yosihiko H. et Marimari J. Kellum, 1965. Preliminary report on excavations in the Marquesas Islands, French Polynesia, Honolulu, B.P. Bishop Museum Polynesian Archaeological Program National Science Foundation Grant., ms, 55 p. 12 fig.
SKJOLSVOLD Arne, 1972. Excavation of a habitation cave, Hanapete'o valley, Hiva Oa, Marquesas Islands, Honolulu, Pacific Anthropological Records 16, Department of Anthropology Bernice P. Bishop Museum.

Smith Carlyle S., 1964. Archaeological Investigations at Pekia, Hiva Oa, Marquesas Islands, Kansas, University of Kansas, Lawrence.

VON DEN Steinen Karl, 1979 (1 ${ }^{\mathrm{e}}$ éd. 1925-1928). Die Marquesaner und ihre Kunst, 3 vol, Berlin, Hacker Art Books.

-, 1997 à 1999. Mythes marquisiens, te hakatu tumu o te ati enana, 3 tomes, Papeete, éditions Haere po no Tahiti.

Solsvik Reidar, 1999. Cultivating an Identity: Agriculture and Social Space in Hanatekua valley, Hiva Oa, Marquesas Island, No barriers seminar papers, vol. 2, The Kon Tiki Museum, pp. 29-32.

Testard De Marans Alfred, 2004. Souvenirs des îles Marquises, groupe Sud-Est, 1887-1888, Paris, Publications de la Société des Océanistes 45. 
